\title{
Cosmologists at odds over mysterious anomalies in data from early Universe
}

Planck satellite's picture of cosmic microwave background needs correction, some researchers argue.

\section{Ron Cowen}

13 December 2013

\section{DALLAS}

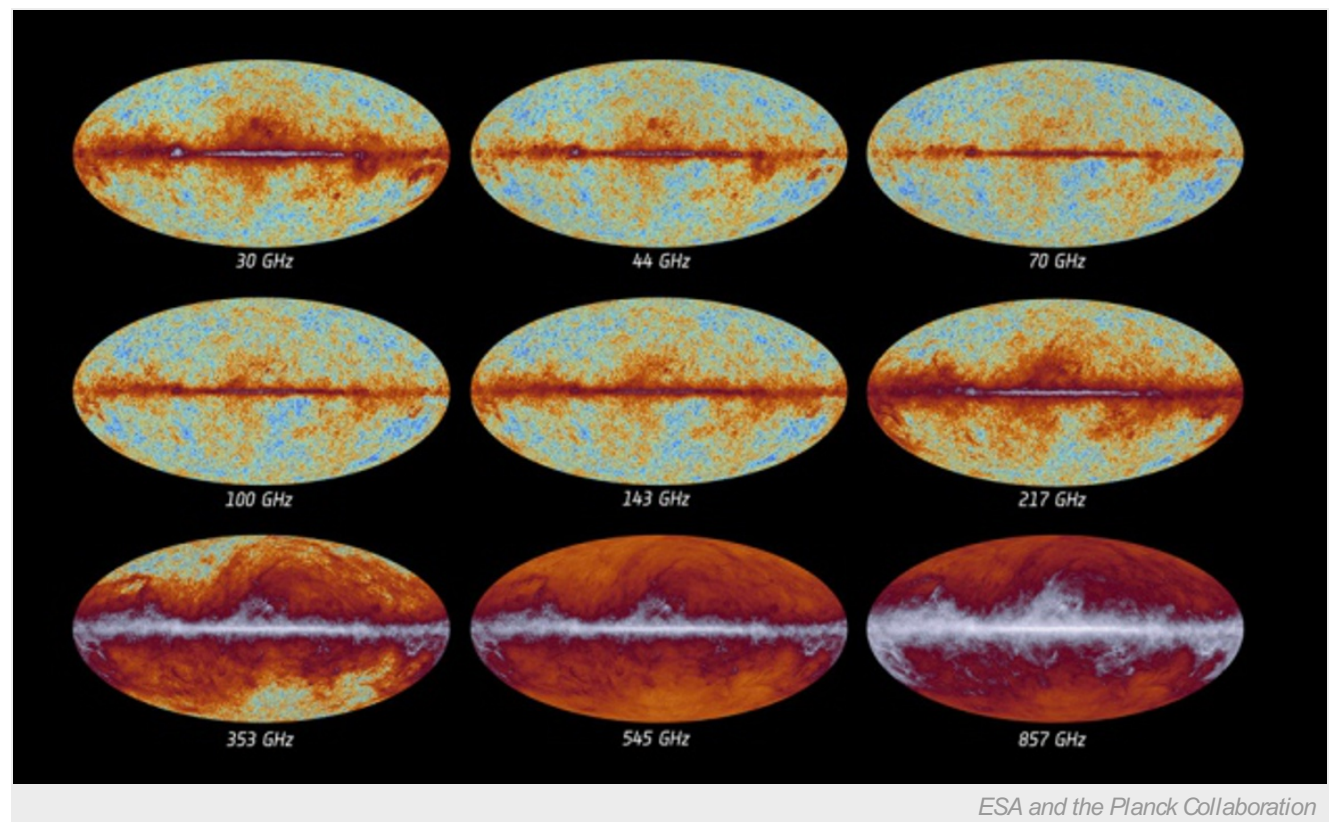

The Planck probe's picture of the full sky — which it took in different bands of the microwave spectrum may have been plagued by systematic errors in the 217-gigahertz band, a new study suggests.

A new analysis of data on the infant universe released earlier this year indicates that the initial findings are more in line with the standard cosmological picture than had originally been thought.

There were both celebrations and surprises last March when the science team of the European Space Agency's Planck mission unveiled a new map of the cosmic microwave background (CMB), the radiation left over from the Big Bang. The precision data formed the highestresolution all-sky CMB map ever. And it revealed a higher density of matter, a slightly greater variation in the distribution of that matter, and a lower value of the Hubble constant - a measure of the Universe's expansion rate — than a host of previous studies from the ground and space had indicated. 
Although the differences were slight, cosmologist David Spergel of Princeton University in New Jersey was intrigued. "Planck is so precise that even small discrepancies become interesting," he notes. The initial findings, he says, suggested one of three possibilities: Either the standard cosmological model might have to be modified; or a host of different astronomical studies were incorrect; or some systematic error in the Planck data had not been accounted for.

A new analysis of the Planck data by Spergel, Renée Hložek of Princeton and Raphael Flauger of the Institute for Advanced Study in Princeton and New York University indicates that the problem lies with the Planck sky map data recorded at a radio frequency of 217 gigahertz. When they removed the 217-GHz data from the maps and relied on two lower frequencies, 100 and $143 \mathrm{GHz}$, the results essentially fell in line with previous CMB and other astrophysical studies, including NASA's Wilkinson Microwave Anisotropy Probe (WMAP). Spergel had led the analysis for that mission.

Spergel presented the findings on 10 December at the 27th Texas Symposium on Relativistic Astrophysics in Dallas. He and his collaborators also posted the study on the arXiv preprint server ${ }^{2}$.

In one of the Planck team's many research papers released in March, the scientists noted that the data recorded at $217 \mathrm{GHz}$ failed some calibration tests ${ }^{1}$. However, the method that the Planck team chose to analyse the data did not account for this issue.

Spergel and his team also compared Planck data recorded during the first half to that taken during the second half of the craft's observing seasons. That comparison revealed that the $217-\mathrm{GHz}$ data were not independent of data gathered at other frequencies. When Spergel's team corrected for the correlation they found, the cosmological properties deduced from the Planck sky maps were also more similar to those calculated from earlier studies. Detectors at different frequencies might not be independent because they are all housed in the same cooling system, Spergel suggests.

The Planck researchers acknowledge that a systematic error might have been at play, and they say that they will now re-examine their own data analysis, which is currently under review at a journal, says Francois Bouchet, a Planck researcher from the Institute of Astrophysics of Paris.

However they also stand by their earlier claim. "We believe that differences in cosmological parameters derived by WMAP and Planck are not due to [the spurious $217 \mathrm{GHz}$ ] feature, but more simply to the improved performance of the Planck data," says ESA's Jan Tauber, who leads the Planck science team and is based in Noordwijk, The Netherlands.

"There is an issue with one set of detectors on Planck, which we were aware of, but didn't properly understand by the time of the 2013 data release," adds George Efstathiou, an astrophysicist at the University of Cambridge, UK, and another member of the Planck science team. "It is a small systematic and has very little impact on the cosmology, but it is a systematic that will be removed from the 2014 data," he says.

"With an experiment as sensitive as Planck, the great challenge is fully understanding all of the systematics," says Spergel. He adds that just as the analysis of WMAP data continued to improve through its nine years of operations, "I anticipate that the Planck 2014 analysis will be an improvement on the Planck 2013 analysis."

Cosmologist Michael Turner of the University of Chicago in Illinois quips, "Precision cosmology is hard; accurate cosmology is even harder."

Nature | doi:10.1038/nature.2013.14368

\section{References}

1. Ade, P. A. R. et al. Preprint available at http://arxiv.org/abs/1303.5076 (2013).

2. Spergel, D., Flauger, R. \& Hožek, R. Preprint available at http://arxiv.org/abs/1312.3313 (2013).

Nature ISSN0028-0836 ESSN 1476-4687

\section{SPRINGER NATURE}

